\title{
Surrogate Endpoints in Health Technology Assessment: An International Review of Methodological Guidelines
}

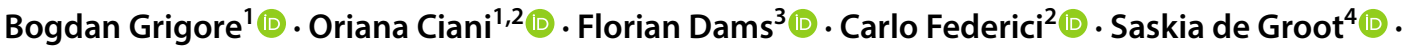

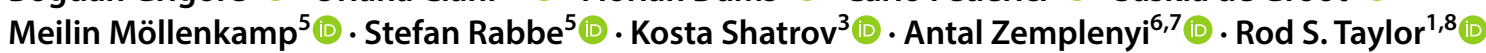

Published online: 23 June 2020

(c) The Author(s) 2020

\begin{abstract}
In the drive towards faster patient access to treatments, health technology assessment (HTA) agencies are increasingly faced with reliance on evidence from surrogate endpoints, leading to increased decision uncertainty. This study undertook an updated survey of methodological guidance for using surrogate endpoints across international HTA agencies. We reviewed HTA and economic evaluation methods guidance from European, Australian and Canadian HTA agencies. We considered how guidelines addressed the methods for handling surrogate endpoints, including (1) level of evidence, (2) methods of validation, and (3) thresholds of acceptability. Across the 73 HTA agencies surveyed, $29(40 \%)$ had methodological guidelines that made specific reference to consideration of surrogate outcomes. Of the 45 methods documents analysed, the majority [27 (60\%)] were non-technology specific, 15 (33\%) focused on pharmaceuticals and three (7\%) on medical devices. The principles of the European network for Health Technology Assessment (EUnetHTA) guidelines published in 2015 on the handling of surrogate endpoints appear to have been adopted by many European HTA agencies, i.e. preference for final patient-relevant outcomes and reliance on surrogate endpoints with biological plausibility and epidemiological evidence of the association between the surrogate and final endpoint. Only a small number of HTA agencies (UK National Institute for Care and Excellence; the German Institute for Medical Documentation and Information and Institute for Quality and Efficiency in Health Care; the Australian Pharmaceutical Benefits Advisory Committee; and the Canadian Agency for Drugs and Technologies in Health) have developed more detailed prescriptive criteria for the acceptance of surrogate endpoints, e.g. meta-analyses of randomised controlled trials showing strong association between the treatment effect on the surrogate and final outcomes. As the decision uncertainty associated with reliance on surrogate endpoints carries a risk to patients and society, there is a need for HTA agencies to develop more detailed methodological guidance for consistent selection and evaluation of health technologies that lack definitive final patient-relevant outcome evidence at the time of the assessment.
\end{abstract}

\section{Background}

A key issue in the increasing move towards early access to new and innovative healthcare technologies is the use of surrogate endpoints to support licensing and coverage decisions of such technologies. Within this context [1,2], a surrogate endpoint is defined as a biomarker (e.g. blood pressure) or an intermediate outcome (e.g. exercise capacity) that can substitute for a final patient-relevant outcome that includes

Electronic supplementary material The online version of this article (https://doi.org/10.1007/s40273-020-00935-1) contains supplementary material, which is available to authorized users.

Bogdan Grigore

b.grigore@exeter.ac.uk

Extended author information available on the last page of the article mortality and health-related quality of life [3]. Disease areas with a strong tradition of surrogate endpoints include oncology (e.g. tumour response for overall survival) and cardiovascular disease (e.g. blood pressure for cardiovascular mortality or morbidity). In clinical areas (e.g. dermatology or acute disease) where patient-relevant outcomes are relatively quickly accrued, the need for surrogate endpoints is much less.

Many regulatory decisions across the world rely on surrogate endpoint evidence. Surrogate endpoints are the primary endpoints in almost half of the studies submitted to the US FDA for marketing approval of medicines [4, 5]. Recently, to inform the development pathways of medicines, the FDA published a list of accepted surrogate endpoints and disease areas that were the basis of approval or licensing of a 


\section{Key Points for Decision Makers}

Although surrogate endpoints enable faster trials and therefore faster access to treatment, they increase the uncertainty of coverage decisions on health technologies

Our survey shows that many international health technology assessment (HTA) agencies currently lack detailed guidance for the evaluation of health technologies that rely on surrogate endpoint evidence

HTA agencies need to provide more detailed and prescriptive guidelines for the consistent qualification and incorporation of surrogate endpoint evidence in the decision processes where the evidence on patient-relevant endpoints is lacking

Current best knowledge suggests that adequate approaches include evidence hierarchy frameworks, meta-regression analytical techniques and economic modelling methods that explicitly explore the uncertainty in the surrogate-to-final endpoint relationship

medicinal or a biological product under both the accelerated and the traditional approval pathways [6].

Whilst surrogate endpoints enable faster outcome accrual and therefore shorter clinical trials [7], reliance on such endpoints can be problematic if they fail to fully capture the complete risk-benefit profile of a health technology [8]. Surrogate endpoints have been shown to overestimate intervention effects [9] and, in some cases, lead to increased risk of harm $[10,11]$.

As the use of surrogate endpoints has become more common in the licensing of new health technologies [12], health technology assessment (HTA) agencies $[12,13]$ are under increasing pressure to utilise such evidence in their recommendations that inform the coverage and funding of medicines and medical devices. Whether surrogate endpoint evidence is used to interpret clinical effect in the context of insufficient final patient-relevant endpoint information [14] or is translated to a different outcome (such as quality-adjusted life-year [QALY]) within an economic model [15], there is a need to ensure that the choice of surrogate is adequate. Therefore, it has been recommended that the use of surrogate endpoints be limited only to those that have been validated appropriately $[1,12,16]$. Such validation ideally requires $(1)$ experimental evidence that demonstrates (2) an acceptable association between treatment-induced change on surrogate endpoint and treatment-induced change on final patient-relevant endpoint and (3) a quantification of the treatment-induced change on final patient-relevant endpoint based on the observed treatment-induced change on surrogate endpoint [1].
In 2009, Velasco Garrido and Mangiapane [17] published a survey of methodological guidance across international HTA agencies. Although 20 of 34 methods guidelines were reported to include surrogate endpoints, the depth and breadth varied considerably between documents. The authors concluded that "the role of surrogate outcomes in HTA is very limited", with many agencies accepting health technologies based on surrogate endpoint evidence in the absence of definitive final endpoint data as exceptional and only when the validity of the surrogate endpoint has been proven. However, few agencies provided details on how such 'validity' would actually be assessed.

Given recent developments in accelerated and adaptive licensing pathways, this study undertook an updated survey to gain a contemporary picture of methods for the handling of surrogate endpoints by international HTA agencies. As this study was conducted within the European Unionfunded COMED (Pushing the boundaries of Cost and Outcome analysis of Medical Technologies) project [18], we also sought to assess whether these methods for handling surrogate endpoints included specific provision for medical device technologies.

\section{Methods}

We sought to identify recommendations on approaching surrogate endpoint evidence in HTA as reflected by current public guidelines and technical documents from relevant HTA bodies.

\subsection{Identification of Health Technology Assessment (HTA) Agencies}

We updated the listing of European HTA agencies from the previous 2009 survey of surrogate endpoints [17] to include all organizations currently listed as members of three major HTA networks (as of March 2018): Health Technology Assessment International, the European network for Health Technology Assessment (EUnetHTA) and the International Network of Agencies for Health Technology Assessment. We included all HTA agencies unless they were patient organisations, organisations whose members/stakeholders were the industry, and university centres, hospitals and professional organisations only involved in the production of HTA reports but not in policy guidance or methods development. Additionally, we included Australian and Canadian HTA agencies as they have been established for many years and therefore reflect 'mature HTA settings', i.e. the Australian Pharmaceutical Benefits Advisory Committee (PBAC), the Australian Medical Services Advisory Committee (MSAC) and the Canadian Agency for Drugs and Technologies in Health (CADTH). For each HTA agency, we checked 
for the publicly available methodological guidance (in any language) either as guidelines or as methodological advisory documents (such as those of the UK National Institute for Health and Care Excellence [NICE] Decision Support Unit [19]). Agencies without available methods guidance were excluded.

\subsection{Document Review and Data Extraction}

We assessed the availability and the detail level of the guidance on the use of surrogate endpoints evidence in HTA processes that was provided by the included HTA organisations. Assessment included (1) terminology (including definitions) on the use of surrogate endpoints, (2) methods of surrogate validation and (3) methodological practices recommended in guidance documents.

\subsection{Stage 1: Identification of HTA Agency Methods Guidance on the Use of Surrogate Endpoints}

Websites of all included HTA agencies were screened using a combination of search terms (HTA, guidelines, methods, resources, publications, surrogate, intermediate and endpoints) to identify methods guidance availability and relevant methods documents. This was supplemented by hand searching of the relevant link categories on the websites. Where necessary, agencies were also contacted directly to enquire about relevant documents. For each included agency, the following data were extracted: (1) name of agency and country, (2) name and website location of the methods document, (3) language of the guideline, (4) text detailing use of surrogate endpoints (including location within the document and any citations referenced), (5) assessment of whether the guidance was specific to pharmaceuticals or medical devices or both and to certain disease areas (e.g. cancer). A data extraction form was developed and piloted by two authors (BG and OC) on a sample including documents in English, French and Italian to test the feasibility of the process and to ensure that captured data were appropriate and sufficient for the study's objective. The revised extraction form was then used by a single reviewer with language skills for each agency (OC, CF, BG, MM, SR, FD, KS, SdG, AZ) between April and July 2018. A random sample of 20 documents was then checked by a second reviewer (OC, BG, SR, SdG). For the purposes of presentation in this report, all text was translated into English.

\subsection{Stage 2: Detailed Analysis of Surrogate Methodological Advice}

For each agency identified in stage 1 as including advice on the use of surrogate endpoints in their methods guidance, a more detailed data analysis framework was applied (see Table 1).

\subsection{Data Analysis and Presentation}

The findings of this survey are presented descriptively and in detailed summary results tables.

\section{Results}

\subsection{Selection of HTA Agencies}

A total of 73 HTA agencies met the inclusion criteria (see Fig. 1; Table 2); 29 were excluded because they had no published methodological guidance. Of the remaining 44 agencies, 29 (66\%) included consideration of the handling of surrogate endpoints in their methods guidance. These 29 agencies included 18 European countries (Austria, Belgium, Bulgaria, Croatia, Germany, Spain, France, Germany, Hungary, Ireland, Italy, Netherlands, Norway, Poland, Portugal, Sweden, Slovakia, United Kingdom), the EUnetHTA network of agencies, and the agencies of PBAC, MSAC and CADTH. In total, 45 methodological guidance documents outlining the use of surrogate endpoints were included for analysis. Sources of these documents are presented in Table S1 in the electronic supplementary material (ESM).

\subsection{Consideration of Surrogate Endpoints}

The extent to which methodological guidelines provided specific consideration on the use of surrogate endpoints varied greatly between agencies. The guidance documents of three (10\%) HTA agencies (the Agency for Quality and Accreditation in Health Care and Social Welfare in Croatia (AAZ), the Galician Agency for Health Technology Assessment in Spain and the Norwegian Institute of Public Health) only mentioned surrogate endpoints in general terms and provided no specific methods guidance on their use.

Reflective of the collaborative partnership in the EUnetHTA project, methods guidance of many agencies was based on the guidance on surrogate endpoint methods published by the EUnetHTA in November 2015 [20]. Table 3 provides a summary of key aspects of the EUnetHTA guidance. Whilst the EUnetHTA guidelines state a preference for using final patient-relevant outcomes rather than surrogate outcomes, they also recognise the need to use surrogate/intermediate outcomes. For example, when evidence of the direct effect of the intervention on patient-relevant outcomes (such as mortality or health-related quality of life) is not available, the EUnetHTA guidelines propose criteria for acceptability of a surrogate endpoint: (1) a biological/ 
Table 1 Domains used to extract data from methods documents in stage 2

\begin{tabular}{ll}
\hline Domain & Explanation \\
\hline $\begin{array}{l}\text { Definition } \\
\text { Examples }\end{array}$ & $\begin{array}{l}\text { Is a definition of surrogate endpoints provided as part of the document? } \\
\text { Are examples of surrogate endpoints provided in the text of the document (e.g. progression-free survival as a } \\
\text { surrogate endpoint for overall survival)? }\end{array}$ \\
$\begin{array}{l}\text { Use of surrogates considered } \\
\text { Are considerations on the use of surrogate endpoints included in the guidelines, such as recommendations to cau- } \\
\text { tion when including surrogate endpoints in the analysis? }\end{array}$ \\
$\begin{array}{l}\text { Acceptability criteria } \\
\text { Evidence strength assessment }\end{array}$ & $\begin{array}{l}\text { Is there a framework for quantifying the evidence on the surrogate-final outcome relationship? Level 1-evi- } \\
\text { dence demonstrating that treatment effects on the surrogate correspond to effects on the patient-related outcome } \\
\text { (from clinical trials); level 2-evidence demonstrating a consistent association between surrogate outcome and } \\
\text { final patient-related outcome (from epidemiological/observational studies); level 3-evidence of the biological } \\
\text { plausibility of the relationship between surrogate outcome and final patient-related outcome (from pathophysi- } \\
\text { ological studies and/or understanding of the disease process) }\end{array}$ \\
Are any validation methods prescribed (e.g. correlation of the effects on the surrogate endpoint and the effects on \\
the clinical endpoint from meta-analysis of randomised trials)? \\
Are accepted cut-off values of the surrogate endpoint-to-final outcome association presented?
\end{tabular}

Fig. 1 Summary of agencies and documents selection. HTA health technology assessment

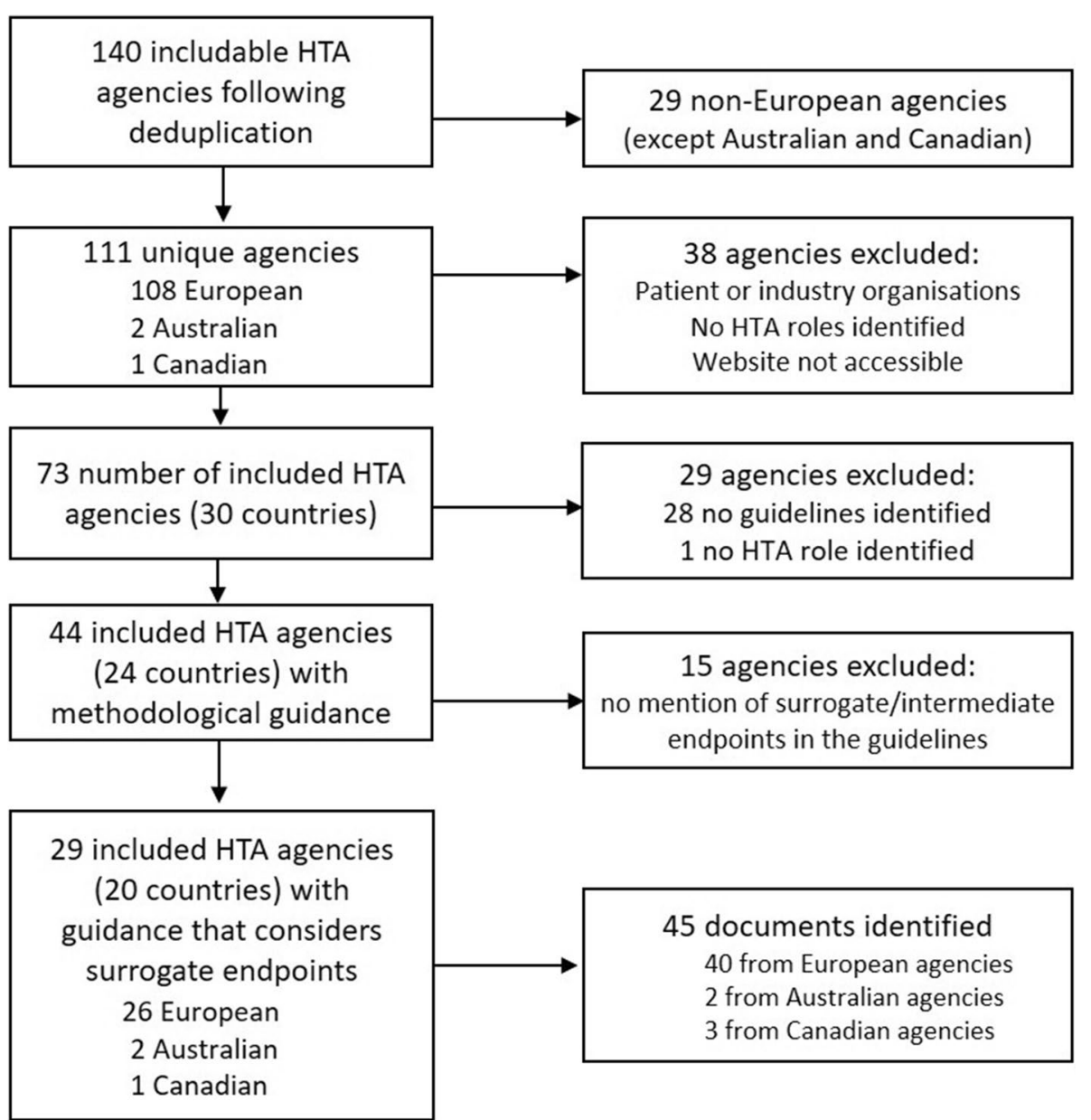

clinical plausibility for the endpoint, (2) evidence of an association with the final patient-relevant endpoint and (3) consideration of wider risk-benefit and/or public health implications [21].

\subsubsection{Definition for Surrogate Endpoints}

In total, 13 methods documents (29\%) provided explicit definitions for surrogate endpoints, many of which were consistent with the EUnetHTA guideline definition, "biomarkers 


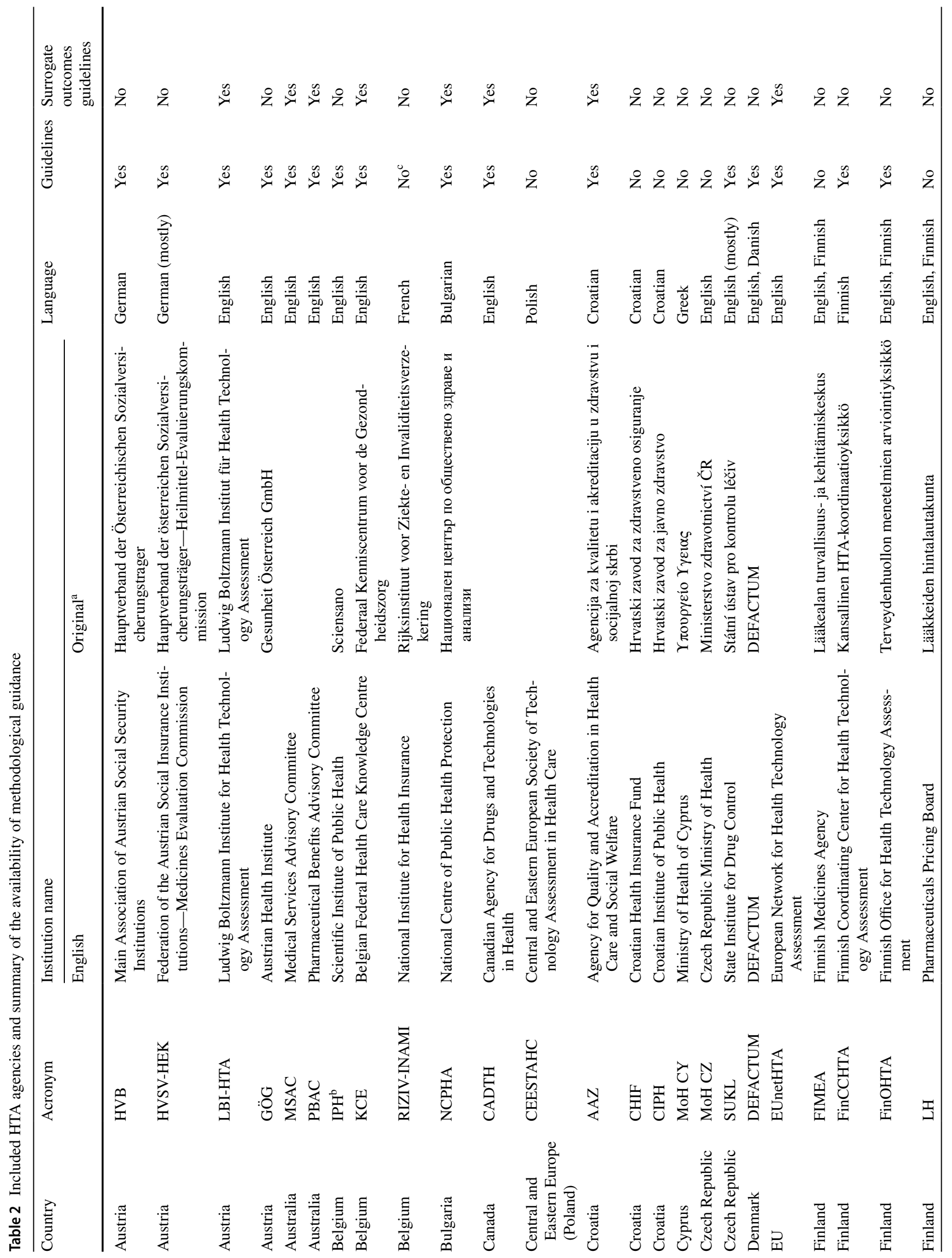




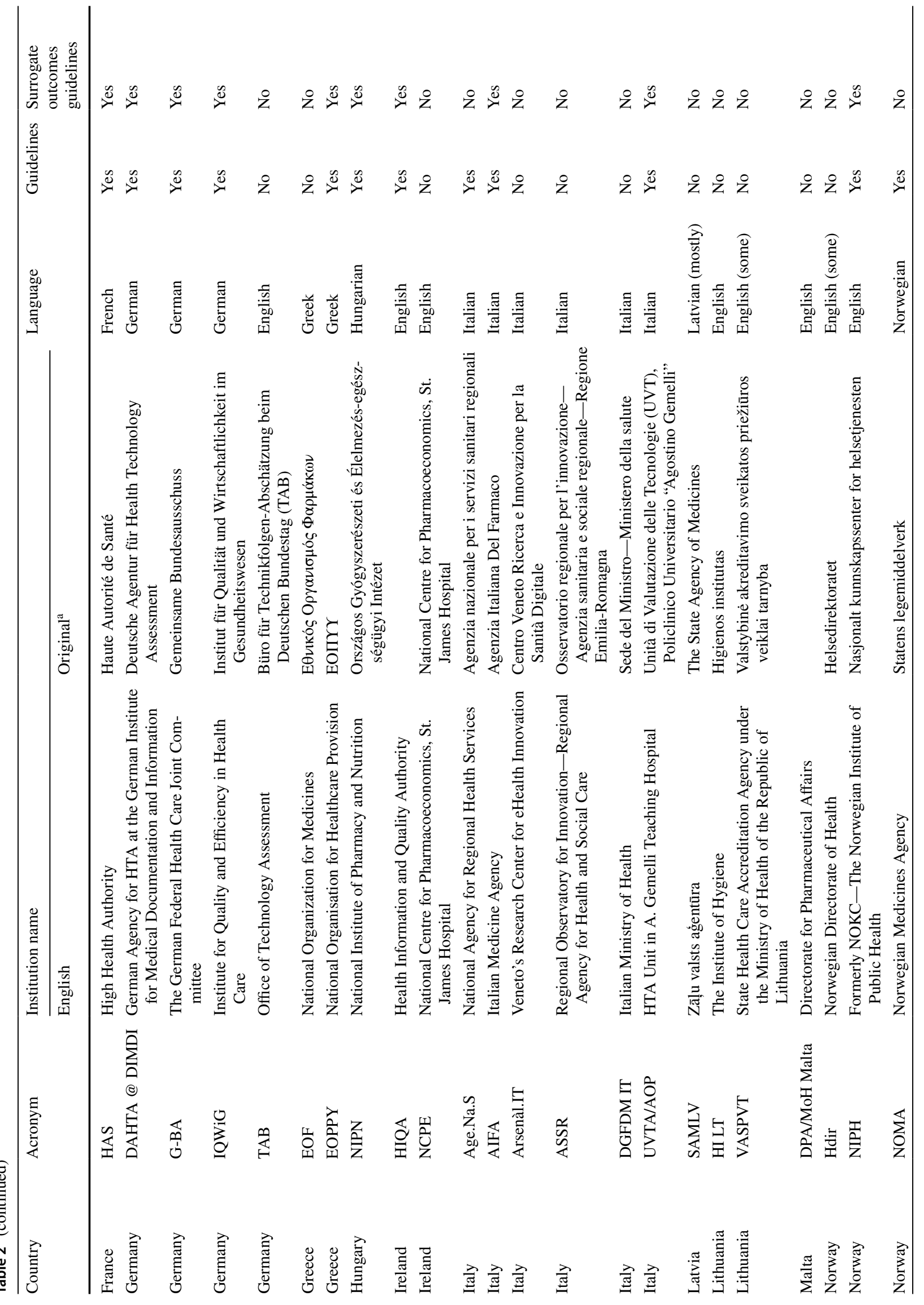




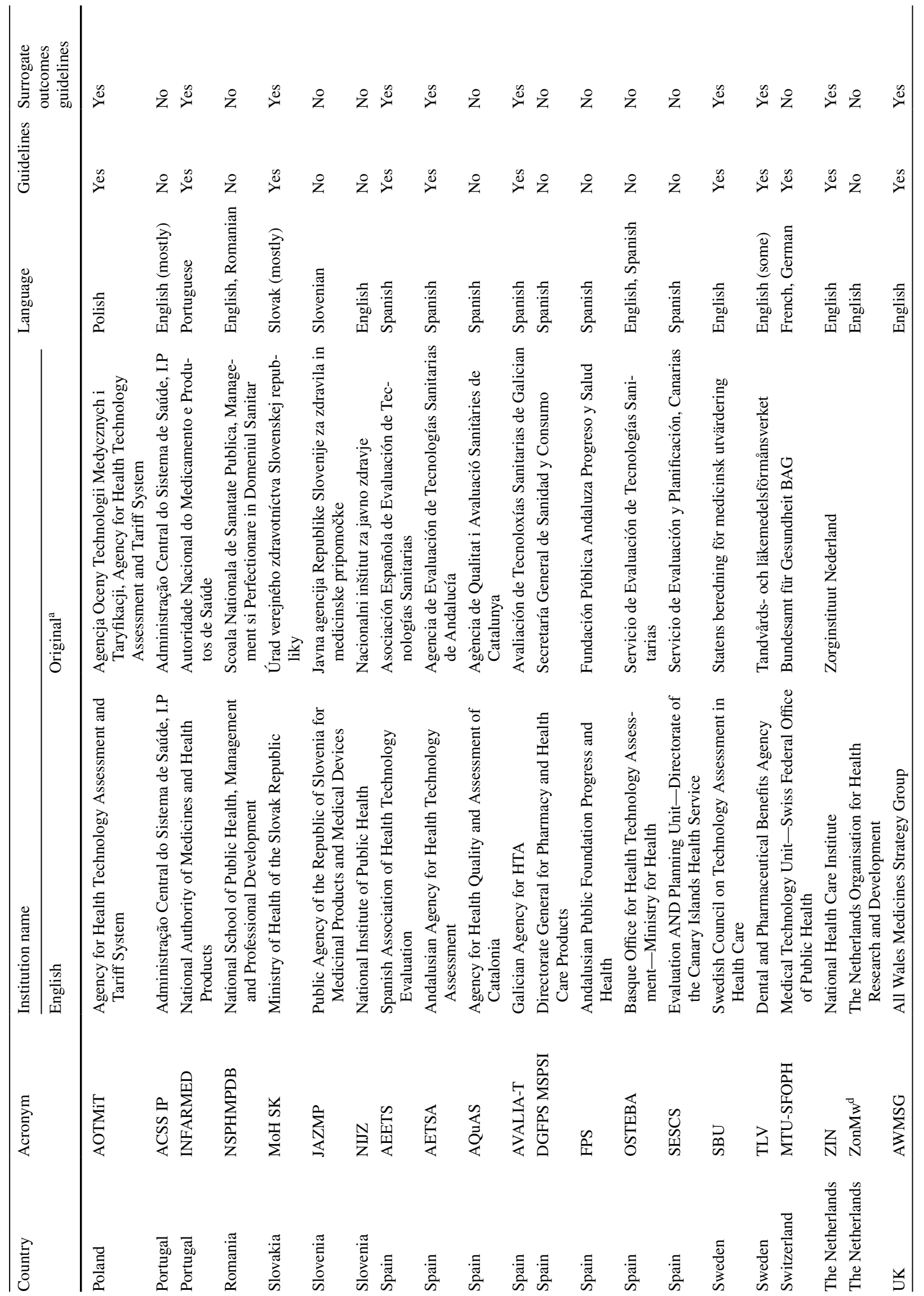




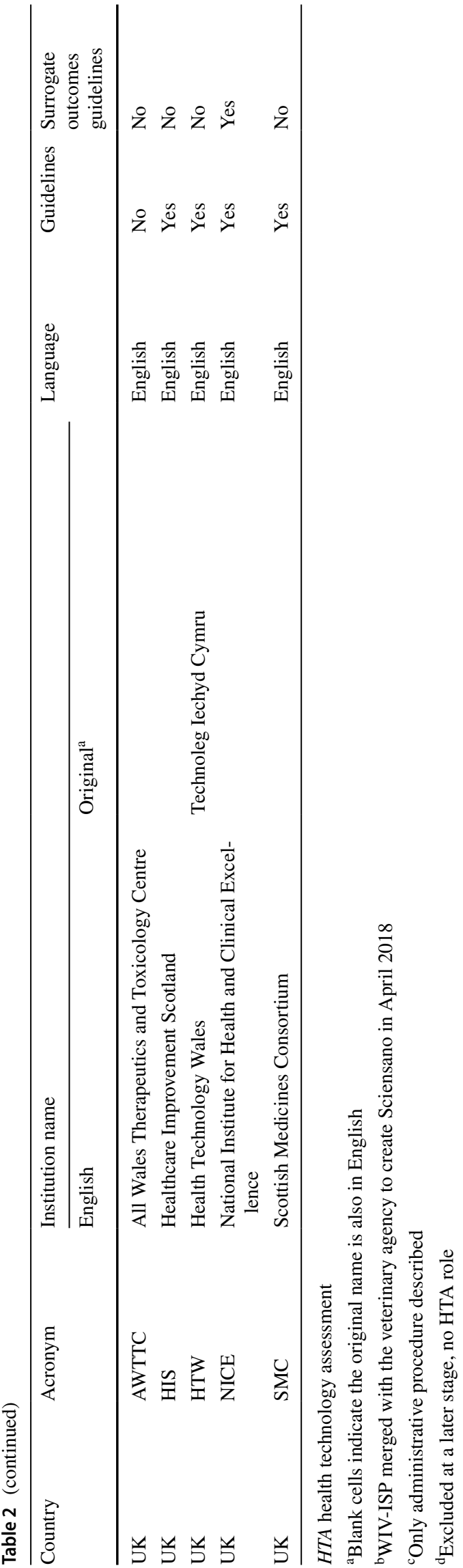

and intermediate endpoints" that can "substitute for a clinically meaningful (final) endpoint". Guidelines from PBAC [22], MSAC [23] and CADTH [24] use similar definitions. For instance, surrogate outcomes are considered by CADTH as "a subset of intermediate outcomes" and are defined as "a laboratory measurement or a physical sign used as a substitute for a clinically meaningful end point that measures directly how a patient feels, functions, or survives" [24]. In their glossary of terms, PBAC defines surrogate outcomes as "a variable that is suspected, but not necessarily demonstrated, to occur on the causal pathway from a clinical management or factor to the clinically relevant final outcome" and recommend the justification and validation of any surrogate outcome used in the analysis [22].

\subsubsection{Example of Surrogate Endpoints}

In total, 18 documents (40\%) provided specific examples of surrogate endpoints (e.g. "Blood pressure as a surrogate endpoint for cardiovascular mortality; bone mineral density as a surrogate for bone fracture; HIV1-RNA viral load as an indicator of viral suppression", Health Information and Quality Authority, Ireland). A support document from the German Institute for Medical Documentation and Information (DIMDI) [25] also provides examples where surrogate endpoints have been proven not to be good surrogates (e.g. increased bone density following treatment of osteoporosis with sodium fluoride did not result in an observed decrease in fractures). A total of 44 documents (98\%) included some consideration of the use of surrogates in the analysis (e.g. "only use a surrogate outcome if it has a well-established link (i.e., validated) with one of (final) outcomes", CADTH). While some guidelines seemed to implicitly consider the surrogate endpoints in a cost-effectiveness context (NICE, PBAC, CADTH), most did not seem to differentiate the interpretation of surrogate endpoints according to the domain (e.g. clinical efficacy, cost effectiveness, etc.). Only four guidelines (from the Austrian Ludwig Boltzmann Institute for Health Technology Assessment, the AAZ, the Polish Agency for Health Technology Assessment and Tariff System (AOTMiT) and CADTH) mentioned the use of surrogate outcomes for safety.

\subsubsection{Acceptability of Surrogate Endpoints}

In total, 26 guidelines (52\%) provided discussion on the acceptability of surrogate endpoints [e.g. "If there is data that validates a surrogate, then these will be assessed in terms of their relevancy and their credibility", German Institute for Quality and Efficiency in Health Care (IQWiG)]. Nine (18\%) clearly refer to the association between surrogate endpoint and final outcome. 
Table 3 Overview of EUnetHTA guidelines for surrogate endpoints

Use of surrogates considered?

Surrogate definition provided?

Examples of surrogates listed?

Acceptability criteria provided?

Evidence strength assessment provided? Yes

"The evidence for the validation of the surrogate-final outcome relationship has been presented by taking into account the level of evidence:

Level 1: evidence demonstrating that treatment effects on the surrogate endpoint correspond to effects on the patient-related clinical outcome (from clinical trials); comprises a meta-analysis of several RCTs and establishment of correlation between effects on the surrogate and clinical endpoint

Level 2: evidence demonstrating a consistent association between surrogate endpoint and final patientrelated endpoint (from epidemiological/observational studies); and

Level 3: only evidence of biological plausibility of relationship between surrogate endpoint and final patient-related endpoint (from pathophysiological studies and/or understanding of the disease process)"

Validation methods provided?

Yes

While the guidelines state that "currently, there is no systematic, transparent and widely agreed-upon process of biomarker validation", they quote correlation of the effects on the surrogate and the effects on the clinical endpoint based on meta-analyses of several RCTs, as well as the surrogate threshold effect [39]

The document offers a selected bibliography addressing the statistical methods of surrogate validation

Validation values provided?
Yes

Although only for information purposes: "There is no clear consensus of which correlation values are sufficient to assume adequate surrogacy, but values of between about 0.85 and 0.95 are often discussed"

HbAlc glycated haemoglobin, ICH International Council for Harmonisation of Technical Requirements for Pharmaceuticals for Human Use, $R C T$ randomised controlled trial

\subsection{Detailed Methodological Guidance on Surrogate Endpoints}

In addition to the EUnetHTA guidelines, seven (15\%) HTA agencies had methods guidance that included detailed methodological consideration of surrogate endpoints: IQWiG (two documents), NICE, AOTMiT, the Portuguese National Authority of Medicines and Health Products (INFARMED), PBAC, MSAC and CADTH. These documents included recommendations of methods to be used for the validation of surrogate endpoints and, in two cases, cut-offs for the acceptance of surrogates according to their validation.

\subsubsection{Methods for Validation of Surrogate Endpoints}

Specific methods recommendations are listed in Table 4. EUnetHTA [20] and IQWiG [26] guidelines are the most detailed and prescriptive European guidelines, providing suggestions of methods for the validation of surrogate outcomes and defining necessary correlation levels for 
the association between surrogate and clinically relevant outcomes [27]. In contrast, NICE technology appraisal guidelines [28] focus on the decision uncertainty associated with evidence and this reflected in the economic modelling of a technology and recommend that "in all cases, the uncertainty associated with the relationship between the end point and health-related quality of life or survival should be explored and quantified" [29]. PBAC guidance [22] contains a supplementary appendix that outlines a prescriptive approach to validating surrogate endpoints for decision modelling based on a four-step approach: (1) identify the surrogate endpoints and the corresponding final outcome; (2) establish the biological plausibility of the two, and present epidemiological evidence to support it; (3) present randomised trial evidence to support the nature of the relationship; (4) translate the treatment effect on the surrogate endpoints to an estimate of the comparative treatment effect for the final outcome [22].

\subsubsection{Specific Guidance for Disease Areas}

In three cases, specific guidance on the use of surrogate endpoints in oncology was available: NICE [30] analysed the suitability of particular surrogate endpoints (such as progression-free survival for overall survival in cancer), and IQWiG [27] provided a detailed discussion on the potential use of surrogate outcomes in oncology. In CADTH guidance, a document dedicated to the evaluation of oncology therapies [31] contained detailed discussion of acceptability of surrogate outcomes according to their correlation with patient outcomes and the treatment intent (curative, adjuvant or palliative).

\subsubsection{Specific Guidance for Medical Devices}

Of the 45 methods documents analysed, 15 (33\%) were exclusively intended for pharmaceuticals, and only three (7\%) were intended exclusively for the evaluation of medical devices (NICE Medical Technology Evaluation Programme (MTEP), the State Institute for Drug Control in the Czech Republic and MSAC). Table 5 provides a comparison of the methods guidelines across HTA programmes aimed at evaluating either general health technologies or pharmaceuticals versus those for evaluating medical devices in the UK (NICE technology appraisal vs. MTEP) and Australia (PBAC vs. MSAC). Guidelines for medical devices appeared less specific and did not include any specific methodological recommendations beyond a general need to provide supporting evidence for surrogate endpoints (Table 5).

\section{Discussion}

Our updated international survey included 74 HTA agencies, of which 29 (39\%) had methodological guidance documents that included consideration of surrogate endpoints. Many of the European agencies' methods guidelines appear to have been revised to reflect the principles of the EUnetHTA guidelines on surrogate endpoints published in 2015 [20]. The EUnetHTA guidelines state a preference for evidence from final patient-relevant outcomes (such as mortality and health-related quality of life) and advise cautious consideration when surrogate endpoints are used, i.e. use of 'validated' surrogate endpoints. However, although the EUnetHTA guidelines are a useful development, they do not provide any explicit criteria to establish whether or not a surrogate endpoint is valid. Furthermore, none of the HTA guidelines in our survey included a list of 'accepted' surrogate endpoints, i.e. surrogate endpoints for which the future use in an evaluation would not require justification.

We identified only five HTA agencies (IQWiG, DIMDI, NICE, PBAC and CADTH) with guidelines providing specific prescriptive methodological advice on the statistical methods that should be used for the validation and assessment of acceptability of surrogate endpoints. Whilst there was a recognition across these guidelines of the lack of methodological consensus around the level of evidence necessary for the validation of surrogates, consensus was strong on the need for randomised trial data to support the association in the treatment effect between surrogate and final endpoints, including the use of meta-regression analysis methods. However, only a IQWiG document currently discusses numerical values for an acceptable level of association (e.g. $R^{2}$ trial $>0.49$ ) [27]. Our results showed little difference in guidance between the use of surrogate endpoints for clinical effectiveness and for incorporation into economic models, with the exception of the NICE technical guidance approach, which focuses on the exploration of uncertainty in the surrogate-to-final-outcome relationship as part of the probabilistic sensitivity analysis. Since our study was conducted, the NICE decision support unit published another technical document [32]. This report focused on the use of multivariate meta-analytic methods for combining data from multiple correlated outcomes for the purpose of surrogate endpoint evaluation and suggested that, instead of criteria about the correlation, it is important to look at predicted estimates and their uncertainty because the strength (or weakness) of the surrogate relationship will manifest itself in the width of the predicted interval of the treatment effect on the final outcome.

The majority of methodological documents on surrogate endpoints identified in our study were intended to be applied 
Table 4 HTA agencies with detailed methods for the handling of surrogate endpoints

\begin{tabular}{|c|c|c|}
\hline Agency & Methods for validation of surrogate endpoints & Cut-offs for the acceptance of surrogate endpoints \\
\hline MSAC (Australia) & $\begin{array}{l}\text { MSAC propose a three-step approach to validate the } \\
\text { transformation of a surrogate endpoint to estimate final } \\
\text { outcomes: } \\
\text { Step } 1 \text { requires a systematic review "to examine whether } \\
\text { epidemiological evidence and biological reasoning } \\
\text { has established that there is a relationship between the } \\
\text { surrogate outcome and the final outcome independent of } \\
\text { any intervention" } \\
\text { Step } 2 \text { requires a systematic review "to examine whether } \\
\text { direct randomised trial evidence using other active } \\
\text { medical services has shown that there is a basis to con- } \\
\text { clude that a treatment effect on the surrogate outcome } \\
\text { has satisfactorily predicted a treatment effect on the } \\
\text { final outcome. [...] Based on this evidence, quantify } \\
\text { the relationship between these treatment effects with an } \\
\text { assessment of the uncertainty of the relationship" } \\
\text { Step } 3 \text { requires an explanation "why this relationship } \\
\text { between the treatment effects on these outcomes with } \\
\text { these other active medical services is likely to apply } \\
\text { to the proposed therapeutic medical service. [...] At } \\
\text { present, it is difficult to give categorical advice" [23] }\end{array}$ & Not reported \\
\hline PBAC (Australia) & $\begin{array}{l}\text { PBAC propose a four-step approach to validating the use } \\
\text { of a surrogate endpoint to predict a final outcome: } \\
\text { "A5.1-Define the PSM and the TCO } \\
\text { A5.2-Establish the biological reasoning for the link } \\
\text { between the PSM and the TCO, including how pivotal } \\
\text { the PSM is to the causation pathway of the TCO, and } \\
\text { present epidemiological evidence to support this } \\
\text { A5.3-Present randomised trial evidence to support the } \\
\text { nature of the PSM-TCO comparative treatment effect } \\
\text { relationship } \\
\text { A5.4-Translate the comparative treatment effect on } \\
\text { the PSM from the studies included in Part A, Subsec- } \\
\text { tion 2.2, to an estimate of the comparative treatment } \\
\text { effect for the TCO." [22] }\end{array}$ & Not reported \\
\hline CADTH (Canada) & $\begin{array}{l}\text { "Validated surrogate outcomes are proven to be predictive } \\
\text { of an important patient outcome. A surrogate outcome } \\
\text { is valid only if there is a "strong, independent, consist- } \\
\text { ent association" with an important patient outcome, } \\
\text { and there is "evidence from randomized trials that ... } \\
\text { improvement in the surrogate end point has consistently } \\
\text { lead to improvement in the target outcome." [24] }\end{array}$ & Not reported \\
\hline DIMDI (Germany) & $\begin{array}{l}\text { No gold standard for the validation of surrogate end- } \\
\text { points, but approaches based on several studies, such as } \\
\text { meta-analyses, are preferred } \\
\text { Regardless of statistical method used for validation, vali- } \\
\text { dation should be considered as technology specific [25] }\end{array}$ & Not reported \\
\hline G-BA (Germany) & $\begin{array}{l}\text { Correlation from meta-analyses between effects on the } \\
\text { surrogate outcome and the final outcome }[39,40] \\
\text { Surrogate Threshold Effect [41] }\end{array}$ & Not reported \\
\hline IQWiG (Germany) & $\begin{array}{l}\text { No 'best' method is defined, but correlation-based } \\
\text { validation is the 'preferred' method, in the sense it has } \\
\text { been most widely used in evaluations. Another option } \\
\text { discussed is the surrogate threshold effect [26] } \\
\text { A support document [27] discusses threshold values } \\
\text { reported in the literature, without enforcing them }\end{array}$ & $\begin{array}{l}\text { "A correlation of } R \geq 0.85 ; R^{2} \geq 0.72 \text { measured at the lower } \\
\text { bound of the } 95 \% \text { percentage interval allows to conclude } \\
\text { that the validation study represents a high reliable result. } \\
\text { This interval } R<0.85 ; R^{2}<0.72 \text { to } R>0.7 ; R^{2}>0.49 \\
\text { represents a medium reliable result between surrogate } \\
\text { and patient-relevant endpoint. If a validation study shows } \\
\text { high reliable results with statistically low correlation } \\
\left(R \leq 0.7 ; R^{2} \leq 0.49\right) \text { measured at the lower bound of the } \\
\text { confidence interval then the surrogate is not considered } \\
\text { as a valid endpoint" [27] }\end{array}$ \\
\hline
\end{tabular}


Table 4 (continued)

\begin{tabular}{|c|c|c|}
\hline Agency & Methods for validation of surrogate endpoints & Cut-offs for the acceptance of surrogate endpoints \\
\hline EUnetHTA & $\begin{array}{l}\text { Correlation from meta-analyses of several RCTs between } \\
\text { the effects on the surrogate and the effects on the clini- } \\
\text { cal endpoint } \\
\text { If there is no high correlation demonstrated, conclusions } \\
\text { might still be made if the surrogate threshold effect is } \\
\text { considered [41] }\end{array}$ & $\begin{array}{l}\text { "Values of between about } 0.85 \text { and } 0.95 \text { are often dis- } \\
\text { cussed" }\end{array}$ \\
\hline AOTMiT (Poland) & $\begin{array}{l}\text { "If the clinical effectiveness assessment is based on the } \\
\text { results of surrogate endpoints, the clinical analysis must } \\
\text { reliably demonstrate their relationship with the clini- } \\
\text { cally significant outcomes. Validation of the surrogate } \\
\text { endpoints should be carried out in relation to the health } \\
\text { problem in question." } \\
\text { Cites EUNetHTA guidelines for methods }\end{array}$ & Not reported \\
\hline INFARMED (Portugal) & $\begin{array}{l}\text { Seems to use the framework proposed by Bucher et al. } \\
\text { [14] } \\
\text { "For a surrogate measure to be validated, the following } \\
\text { questions should be positively answered: } \\
\text { 1. Does a strong, consistent and independent association } \\
\text { exist between the surrogate outcome and the clinically } \\
\text { relevant outcome? This criterion is necessary but not } \\
\text { sufficient in itself; } \\
\text { 2. Are there any randomized studies on the same class } \\
\text { of medicines, where improvements in the surrogate } \\
\text { outcomes corresponded to improvements in clinically } \\
\text { relevant outcomes? } \\
\text { 3. Are there any randomized studies on different classes } \\
\text { of medicines, where improvements in the surrogate } \\
\text { outcomes corresponded to improvements in clinically } \\
\text { relevant outcomes for the patient?" }\end{array}$ & $\begin{array}{l}\text { To be considered as validated, a surrogate outcome must } \\
\text { comply with criteria from } 1 \text { to } 3 \text {. Verification of these } \\
\text { criteria usually requires a meta-analysis of randomized } \\
\text { studies }\end{array}$ \\
\hline NICE (UK) & $\begin{array}{l}\text { Guidelines are not specific on the validation methods but } \\
\text { emphasise that "in all cases, the uncertainty associated } \\
\text { with the relationship between the end point and health- } \\
\text { related quality of life or survival should be explored and } \\
\text { quantified" }\end{array}$ & Not reported \\
\hline
\end{tabular}

CADTH Canadian Agency for Drugs and Technologies in Health, DIMDI Institute for Medical Documentation and Information, EUnetHTA European network for Health Technology Assessment, G-BA The German Federal Health Care Joint Committee, IQWiG Institute for Quality and Efficiency in Health Care, MSAC Medical Services Advisory Committee, NICE National Institute for Care and Excellence, PBAC Pharmaceutical Benefits and Advisory Committee, PSM proposed surrogate measure, RCT randomised controlled trial, TCO target clinical outcome

across health technologies (medicine, medical device or others) and across medical conditions. Given that the development and use of surrogate endpoints has become particularly common in oncology [33, 34], NICE, IQWiG and CADTH have published specific support documents for the use of surrogates in this clinical area [27, 30, 31]. Commonly used surrogate endpoints for the final outcome of overall survival in cancer include progression-free survival, disease-free survival and tumour response.

Pharmaceuticals and medical devices traditionally have different regulatory and evidence-generation pathways [35]. Given that various countries/agencies have separate HTA processes for the evaluation of medicines and medical devices, we could compare their methodological approaches to the consideration of surrogate endpoints [36]. The NICE technology appraisal is applicable to all medical technologies, whereas the NICE MTEP specifically considers medical devices and diagnostics. Similarly, in Australia, PBAC assesses pharmaceuticals and MSAC assesses medical devices. The PBAC and MSAC guidance on surrogate endpoints was similar, but we found more of a difference within NICE programmes. The NICE technology appraisal programme was much more detailed and directive in guidance than the MTEP, reflecting the traditionally greater evidence requirements for medicines than for devices. Whilst it might be expected that the evidence requirements for the use and validation of surrogate endpoints should not necessarily differ between health technologies and across disease areas, we recognise there may be challenges in application. For example, given the current regulatory requirements, for specific medical devices, randomised controlled trial (RCT) (and sometimes, non-RCT)-level evidence may not be available at the time of HTA appraisal and even after it [35, 37]. It is likely that the requirement of 'several RCTs' for good 


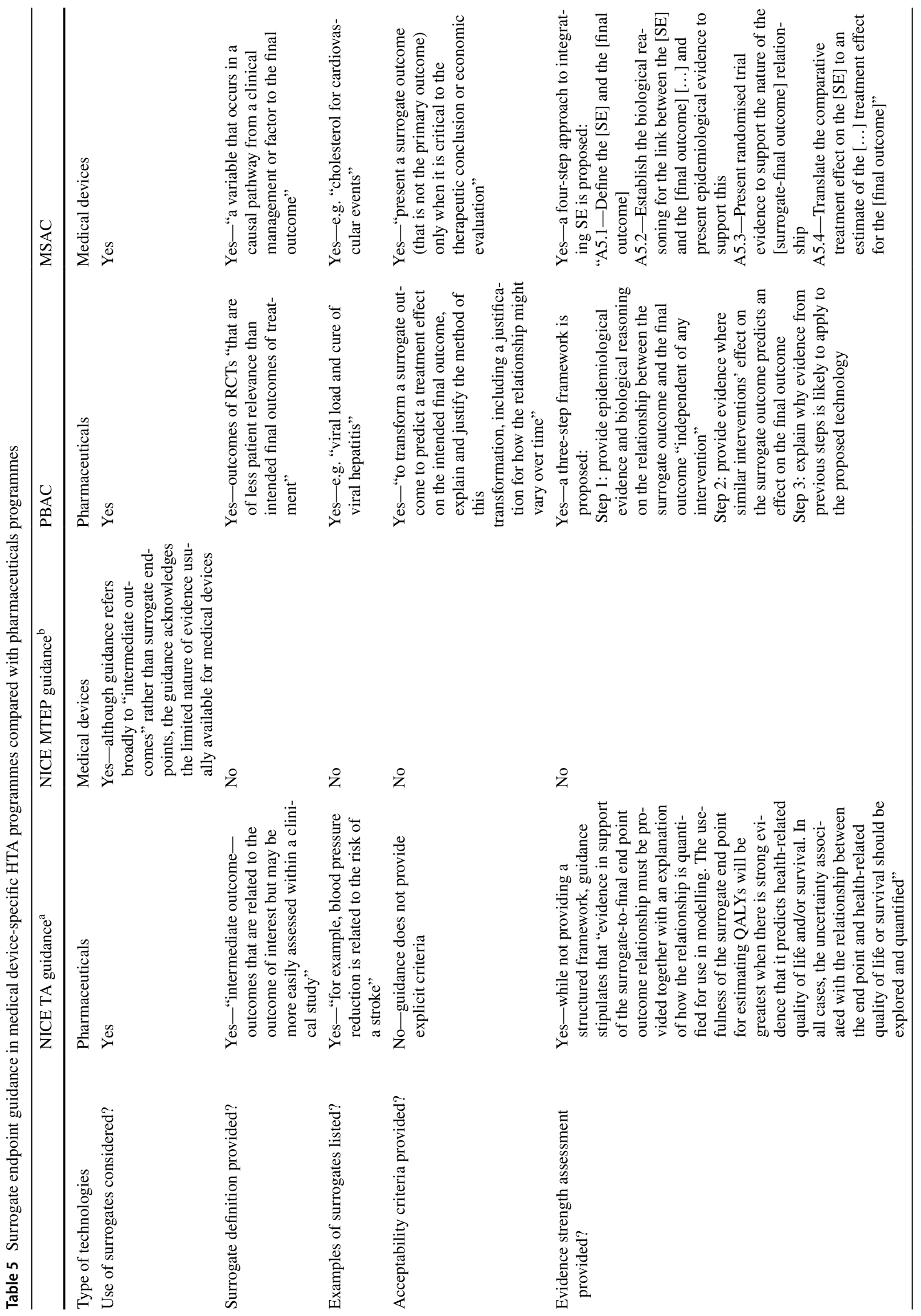


surrogate validation studies will never be satisfied for many indications requiring medical device-based procedures. When confronted with this challenge, there is a temptation to extrapolate validated surrogate endpoints from RCTs of medicines (e.g. the use of systolic blood pressure from RCTs of antihypertensive medicines) to medical-devicebased therapies (e.g. renal denervation therapy). However, we caution against this approach, given that different modes of action and classes of therapies are known to affect the surrogate-to-final-outcome relationship.

Our study provides a comprehensive contemporary review of methods guidance across international HTA agencies on the use of surrogate endpoints. We explored a larger sample of agencies and documents than did the previous survey [17]. However, available resources (particularly time and linguistic access) limited the inclusion of non-European agencies to those of Australia and Canada. Furthermore, this survey only looked at publicly available documents and not at internal documentation that may be circulated within HTA agencies. As described in Sect. 2, methodological advisory documents [27, 30] were also considered, as-in our opinion-they constitute important material to inform and complement methods practice.

\section{Conclusion}

This updated survey of international HTA agencies demonstrates an increase in the methodological guidance for the use of surrogate endpoints over the last decade, largely based on the adoption of EUnetHTA guidance on surrogates published in 2015. Nevertheless, we found considerable differences in the depth of this guidance, with only a few agencies currently having guidelines that provide detailed methodological advice on the statistical methods and metrics for surrogate validation that are deemed acceptable. Further methodological and policy research in the harmonization of approaches to surrogate outcomes evidence in healthcare decision making is warranted. The recent EU proposal of joint HTA clinical assessment [38] may provide the opportunity for implementation of a harmonised approach to the validation of the handling of surrogate endpoints across Europe. Our study also suggests an almost exclusive consideration of surrogate endpoints from a clinical efficacy/ effectiveness perspective. Opportunities therefore remain to further clarify the effective and consistent use of surrogate endpoints in other HTA domains, especially safety and cost effectiveness.

Acknowledgements The authors thank Maria Chaita, Marina Kaisa and Loukia Mantzali for the support provided in the preparation of this manuscript. Oriana Ciani thanks the Fulbright Commission; this manuscript was completed during her research visiting at Yale University. 
Author Contributions Bogdan Grigore contributed to the conception, design of the study and drafting of the protocol, oversaw stage 1 and 2 data extraction and the analysis and interpretation of data and wrote the first draft of the manuscript. Oriana Ciani developed the concept, contributed to the drafting of the protocol, participated in stage 1 and 2 of data extraction and the analysis and interpretation of data and drafted the first version of the manuscript. Florian Dams, Carlo Federici, Saskia de Groot, Meilin Möllenkamp, Stefan Rabbe, Kosta Shatrov and Antal Zemplenyi each reviewed the protocol and conducted stage 1 data extraction and analysis and contributed to the interpretation of data. Rod S Taylor is the guarantor of the study; he developed the concept and participated in the drafting of the protocol and assisted with interpretation of data. All authors agreed on the final version and to each revision of the manuscript.

Data Availability The data that support the findings of this study are available within the article or its supplementary materials.

\section{Compliance with Ethical Standards}

Funding This project received funding from the European Union's Horizon 2020 research and innovation programme under grant agreement \#779306 (COMED—Pushing the Boundaries of Cost and Outcome Analysis of Medical Technologies). The results reflect only the authors' views, and the EU is not responsible for any use that may be made of the information it contains.

Conflict of interest Bogdan Grigore, Oriana Ciani, Florian Dams, Carlo Federici, Saskia de Groot, Meilin Möllenkamp, Stefan Rabbe, Kosta Shatrov, Antal Zemplenyi, and Rod S Taylor have no conflicts of interest that are directly relevant to the content of this article.

Open Access This article is licensed under a Creative Commons Attribution-NonCommercial 4.0 International License, which permits any non-commercial use, sharing, adaptation, distribution and reproduction in any medium or format, as long as you give appropriate credit to the original author(s) and the source, provide a link to the Creative Commons licence, and indicate if changes were made. The images or other third party material in this article are included in the article's Creative Commons licence, unless indicated otherwise in a credit line to the material. If material is not included in the article's Creative Commons licence and your intended use is not permitted by statutory regulation or exceeds the permitted use, you will need to obtain permission directly from the copyright holder. To view a copy of this licence, visit http://creativecommons.org/licenses/by-nc/4.0/.

\section{References}

1. Ciani O, Buyse M, Drummond M, Rasi G, Saad ED, Taylor RS. Time to review the role of surrogate end points in health policy: state of the art and the way forward. Value Health. 2017;20(3):487-95.

2. International Society for Pharmacoeconomics and Outcomes Research. ISPOR 2018 Top 10 HEOR Trends: International Society for Pharmacoeconomics and Outcomes Research; 2018.

3. De Gruttola VG, Clax P, DeMets DL, Downing GJ, Ellenberg SS, Friedman L, et al. Considerations in the evaluation of surrogate endpoints in clinical trials: summary of a National Institutes of Health workshop. Contemp Clin Trials. 2001;22(5):485-502.

4. Downing NS, Aminawung JA, Shah ND, Krumholz HM, Ross JS. Clinical trial evidence supporting FDA approval of novel therapeutic agents, 2005-2012. JAMA. 2014;311(4):368-77.
5. Zhang AD, Puthumana J, Downing NS, Shah ND, Krumholz HM, Ross JS. Assessment of clinical trials supporting US Food and Drug Administration approval of novel therapeutic agents, 1995-2017. JAMA Netw Open. 2020;3(4):e203284. https://doi. org/10.1001/jamanetworkopen.2020.3284.

6. Table of Surrogate Endpoints That Were the Basis of Drug Approval or Licensure [database on the Internet]; 2018. https:// www.fda.gov/Drugs/DevelopmentApprovalProcess/Developmen tResources/ucm613636.htm. Accessed 19 Oct 2018.

7. Hughes MD, Daniels MJ, Fischl MA, Kim S, Schooley RT. CD4 cell count as a surrogate endpoint in HIV clinical trials: a metaanalysis of studies of the AIDS Clinical Trials Group. Aids. 1998;12(14):1823-32.

8. Fleming TR, DeMets DL. Surrogate end points in clinical trials: are we being misled? Ann Intern Med. 1996;125(7):605-13.

9. Ciani O, Buyse M, Garside R, Pavey T, Stein K, Sterne JA, et al. Comparison of treatment effect sizes associated with surrogate and final patient relevant outcomes in randomised controlled trials: meta-epidemiological study. BMJ. 2013;346:f457.

10. Gøtzsche PC, Liberati A, Torri V, Rossetti L. Beware of surrogate outcome measures. Int J Technol Assess Health Care. 1996;12(2):238-46.

11. Rodríguez-Gutiérrez R, Montori VM. Glycemic control for patients with type 2 diabetes mellitus: our evolving faith in the face of evidence. Circulation. 2016;9(5):504-12. https://doi. org/10.1161/CIRCOUTCOMES.116.002901.

12. Kemp R, Prasad V. Surrogate endpoints in oncology: when are they acceptable for regulatory and clinical decisions, and are they currently overused? BMC Med. 2017;15(1):134

13. Breckenridge A, Woods $\mathrm{K}$, Walley $\mathrm{T}$. Medicines regulation and health technology assessment. Clin Pharmacol Ther. 2010;87(2):152-4.

14. Bucher HC, Guyatt GH, Cook DJ, Holbrook A, McAlister FA, Group E-BMW. Users' guides to the medical literature: XIX. Applying clinical trial results A. How to use an article measuring the effect of an intervention on surrogate end points. JAMA. 1999;282(8):771-8.

15. Bulsei J, Darlington M, Durand-Zaleski I, Azizi M. How to perform a cost-effectiveness analysis with surrogate endpoint: renal denervation in patients with resistant hypertension (DENERHTN) trial as an example. Blood Press. 2018;27(2):66-72. https://doi. org/10.1080/08037051.2017.1394160.

16. Buyse M, Sargent DJ, Grothey A, Matheson A, De Gramont A. Biomarkers and surrogate end points - the challenge of statistical validation. Nat Rev Clin Oncol. 2010;7(6):309.

17. Velasco Garrido M, Mangiapane S. Surrogate outcomes in health technology assessment: an international comparison. Int J Technol Assess Health Care. 2009;25(3):315-22.

18. Pushing the boundaries of Cost and Outcome analysis of Medical Technologies (COMED); 2018.

19. National Institute for Health and Care Excellence (NICE). Decision Support Unit. University of Sheffield; 2019. https://niced su.org.uk/. Accessed 15 March 2019.

20. EUnetHTA. Endpoints used for relative effectiveness assessment: Clinical Endpoints; 2015.

21. EUnetHTA. What is Health Technology Assessment (HTA). European Network for Health Technology Assessment. https://www. eunethta.eu/about-us/faq\#t287n73. Accessed 15 Feb 2015.

22. Pharmaceutical Benefits Advisory Committee. Guidelines for preparing submissions to the Pharmaceutical Benefits Advisory Committee. Australian Government, Department of Health and Ageing. 2016.

23. Medical Services Advisory Committee. Technical Guidelines for preparing assessment reports for the Medical Services Advisory Committee-Medical Service Type: Therapeutic (Version 2.0); 2016. 
24. Assessment CCOfHT. Guidelines for economic evaluation of pharmaceuticals: Canada. Canadian Coordinating Office for Health Technology Assessment; 1997.

25. Mangiapane S, Garrido MV. Surrogatendpunkte als Parameter der Nutzenbewertung. Schriftenreihe Health Technology Assessment (HTA) in der Bundesrepublik Deutschland Fachgebiet Management im Gesundheitswesen Berlin: Technische Universität Berlin; 2009.

26. Institut für Qualität und Wirtschaftlichkeit im Gesundheitswesen (IQWiG). Allgemeine Methoden Version 5.0. Version; 2017.

27. Institut für Qualität und Wirtschaftlichkeit im Gesundheitswesen (IQWiG). Aussagekraft von surrogatendpunkten in der onkologie. [Validity of surrogate parameters in oncology (Rapid report). IQWiG-Berichte 80; 2011.

28. National Institute for Health and Care Excellence (NICE). Guide to the methods of technology appraisal 2013. Process and methods. London; 2013.

29. Claxton K, Sculpher M, McCabe C, Briggs A, Akehurst R, Buxton M, et al. Probabilistic sensitivity analysis for NICE technology assessment: not an optional extra. Health Econ. 2005;14(4):339-47.

30. Davis S, Tappenden P, Cantrell A. A review of studies examining the relationship between progression-free survival and overall survival in advanced or metastatic cancer. Technical Support Documents. London: National Institute for Health and Care Excellence (NICE); 2012.

31. Mittmann N, Evans W, Rocchi A, Longo C, Au H, Husereau D, et al. Addendum to CADTH's guidelines for the economic evaluation of health technologies: specific guidance for oncology products. Ottawa: Canadian Agency for Drugs and Technologies in Health; 2009.

32. Bujkiewicz S, Achana F, Papanikos T, Riley R, Abrams K. Multivariate meta-analysis of summary data for combining treatment effects on correlated outcomes and evaluating surrogate endpoints.
Technical Support Documents. London: National Institute for Health and Care Excellence (NICE); 2019.

33. Beaver JA, Howie LJ, Pelosof L, Kim T, Liu J, Goldberg KB, et al. A 25-year experience of us food and drug administration accelerated approval of malignant hematology and oncology drugs and biologics: a review accelerated approvals for malignant hematology and oncology products accelerated approvals for malignant hematology and oncology products. JAMA Oncol. 2018;4(6):849-56. https://doi.org/10.1001/jamaoncol.2017.5618.

34. Ruof J, Knoerzer D, Dünne A-A, Dintsios C-M, Staab T, Schwartz FW. Analysis of endpoints used in marketing authorisations versus value assessments of oncology medicines in Germany. Health Policy. 2014;118(2):242-54.

35. Ciani O, Wilcher B, van Giessen A, Taylor RS. Linking the regulatory and reimbursement processes for medical Devices: the need for integrated assessments. Health Econ. 2017;26:13-29.

36. Campbell G. Statistics in the world of medical devices: the contrast with pharmaceuticals. J Biopharm Stat. 2007;18(1):4-19.

37. Drummond M, Griffin A, Tarricone R. Economic evaluation for devices and drugs-same or different? Value Health. 2009;12(4):402-4.

38. Proposal of Regulation of the European Parliament and of the Council on health technology assessment and amending Directive 2011/24/EU, European Parliament and European Council; 2018.

39. Burzykowski TE. The evaluation of surrogate endpoints. Statistics for biology and health. New York: Springer; 2005.

40. Molenberghs G, Burzykowski T, Alonso A, Assam P, Tilahun A, Buyse M. A unified framework for the evaluation of surrogate endpoints in mental-health clinical trials. Stat Methods Med Res. 2010;19(3):205-36.

41. Burzykowski T, Buyse M. Surrogate threshold effect: an alternative measure for meta-analytic surrogate endpoint validation. Pharm Stat. 2006;5(3):173-86.

\section{Affiliations}

\section{Bogdan Grigore ${ }^{1}\left(\mathbb{D} \cdot\right.$ Oriana Ciani $^{1,2}(\mathbb{D}) \cdot$ Florian Dams $^{3}\left(\mathbb{D} \cdot\right.$ Carlo Federici $^{2}(\mathbb{1}) \cdot$ Saskia de Groot ${ }^{4}(\mathbb{D} \cdot$

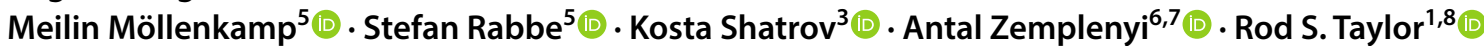

1 Evidence Synthesis and Modelling for Health Improvement, College of Medicine and Health, Institute of Health Research, University of Exeter, Exeter, UK

2 Center for Research on Health and Social Care Management, SDA Bocconi, Milan, Italy

3 KPM Center for Public Management, University of Bern, Bern, Switzerland

4 Institute for Medical Technology Assessment, Erasmus School of Health Policy and Management, Erasmus University Rotterdam, Rotterdam, The Netherlands
5 Hamburg Center for Health Economics, Universität Hamburg, Hamburg, Germany

6 Syreon Research Institute, Budapest, Hungary

7 Division of Pharmacoeconomics, Faculty of Pharmacy, University of Pécs, Pécs, Hungary

8 MRC/CSO Social and Public Health Sciences Unit and Robertson Centre for Biostatistics, Institute of Health and Well Being, University of Glasgow, Glasgow, Scotland 